\title{
Research on the relationship between the age of blood stains and the bending rigidity of blooded cotton fabric
}

\author{
Yichao Cao ${ }^{1,2}$, Kaijun Ma1, Xuejun Zhao ${ }^{1,2}$, Hailou Wang ${ }^{3}$, Fujun Wang ${ }^{3}$, \\ Baozhong Sun ${ }^{3, a *}$ \\ 1 Shanghai Key Laboratory of Crime Scene Evidence, Shanghai, China \\ 2 Shanghai Research Institute of Criminal Science and Technology, Shanghai, China \\ 3 College of Textiles, Donghua University, Shanghai, China \\ sunbz@dhu.edu.cn
}

Keywords: bending rigidity, blood stains, age determination, cotton fabric

Abstract. Blood stains often appear at the scene of the crime. The age determination of blood stains can provide important information to speculate the time of crime. The relationship between the age of blood and the bending rigidity of blooded cotton fabric was investigated experimentally via the use of FAST-2 bending tester. Measure the bending length of blooded fabric at different time and calculate the bending rigidity, then the relationship between the age of blood and the bending rigidity is obtained through curve fitting. The result shows that the bending rigidity of blooded fabric is various with the increase in the age of blood stains and this relationship can be used to speculate the age of blood stains.

\section{Introduction}

Blood stains can be crucial traces and provide important information at crime scenes. The age determination of blood stains in many forensic investigations can be used to determine the moment when a crime was committed, which facilitates detecting a criminal case.

Many approaches have been explored for the age determination of blood stains, including hemoglobin derivative determination[1], hyperspectral imaging[2], relative RNA ratios method[3], atomic force microscopy method[4]. However, sometimes these methods are not feasible to operate.

Bending rigidity is an important mechanical performance of fabric, which reflects the ability to resistant deformation. The bending rigidity of fabric is used to evaluate the fabric style and can also be used to provide clues to detect a criminal case.

The bending rigidity of fabric is related to the fiber bending rigidity, yarn density, count and finishing conditions[5]. In addition, the friction effect between yarns and fibers also influences the bending rigidity of fabric. The difference of friction leads to the different interaction among yarns and fibers, so the bending rigidity of fabric is different.

The blood spreads quickly and is absorbed into yarns and fibers when blood is spattered on fabric, and the bending rigidity of yarns and fiction between yarns is changed, so the bending rigidity of fabric with blood is different. In addition, the bending rigidity of fabric with blood changes continuously over time because the blood changes. In this paper, the influence of blood on the bending rigidity of cotton woven fabric was investigated experimentally. It was aimed that the age determination of blood stains can be speculated through the relationship between the bending rigidity of fabric and the age of blood under controlled environmental conditions. Hope it can be a clue to determine the age of blood in some special cases like a crime case.

\section{Materials and specimens}

In this paper, the cotton plain woven was used as specimens and the specifications of the fabric are shown in Table 1. 
Table 1. Specifications of fabric

\begin{tabular}{|c|c|}
\hline Fabric structure & Plain woven \\
\hline Warp/weft linear density & $20[$ tex $]$ \\
\hline Warp/weft density & $300[$ yarns $/ 10 \mathrm{~cm}]$ \\
\hline Warp tightness & $47.3 \%$ \\
\hline Weft tightness & $39.4 \%$ \\
\hline Total tightness & $68.1 \%$ \\
\hline Weight per unit area & $136.5\left[\mathrm{~g} / \mathrm{m}^{2}\right]$ \\
\hline
\end{tabular}

The pig blood was used in this paper. The blood was drawn on the site and then spattered on the fabric randomly. Considering the blood is easy to dry, the process of spattering blood must be fast. Figure 1 is the fabric after spattered blood.

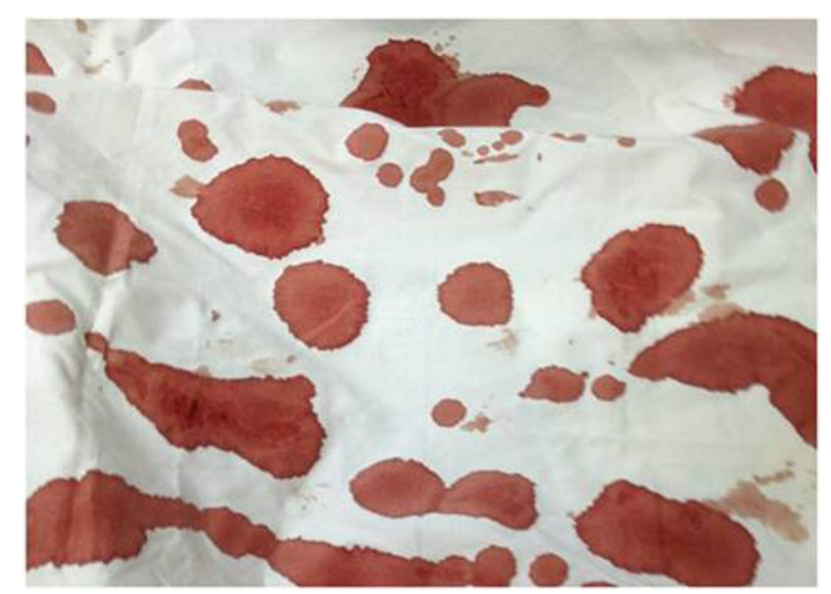

Figure 1. Fabric after spattered blood

\section{Experimental apparatus}

Peirce's cantilever test methodology is preferred to determine the bending stiffness in fabric materials and a fixed angle $41.5^{\circ}$ is used for a inclined surface[6]. The FAST-2 bending tester, which is based on the principle of cantilever test method, was used to test the bending properties of fabric. Under the standard atmosphere enviroment, the FAST-2 bending tester can directly measure the bending length of fabric and then the bending rigidity is calculated from the Equation $(1)[6,7]$.

$$
\begin{aligned}
& \mathbf{B}=w \times \mathbf{C}^{3} \times 9.807 \times 10^{-6} \\
& \text { B: Bending rigidity, }[\mu \mathrm{N} \cdot \mathrm{m}] \\
& \text { w: Weight per unit area, }\left[\mathrm{g} / \mathrm{m}^{2}\right] \\
& \text { C: Bending length, }[\mathrm{mm}] 。
\end{aligned}
$$

The principle of the cantilever test method is showed in Figure 2. FAST-2 bending tester uses phototube to test the bending length and the measure precision is very high. The specimen is moved forward on a low-friction platform and the specimen bends under its own weight. When the specimen touches the $41.5^{\circ}$ angle detectors, the test is completed and the bending length is tested.
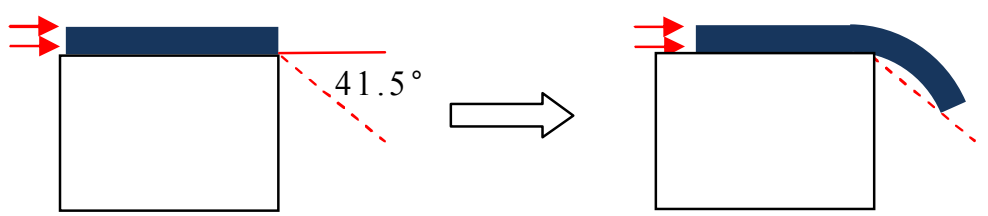

Figure 2. Principle of the cantilever test method used on FAST-2 bending tester 


\section{Experimental procedure}

Put the fabric under the standard atmosphere environment for enough time to balance the fabric before making specimens. Draw fresh pig blood and spatter the blood on fabric randomly, and then take $5[\mathrm{~cm}] \times 20[\mathrm{~cm}]$ spcimens along the warp and weft directions respectively. Measure the warp and weft bending rigidity of specimens on the FAST-2 bending tester at a certain time.

Test time [hour]: 0, 1, 2, 5, 8, 12, 24, 48, 120, 240, 720, 1440, 2160.

\section{Results and discussion}

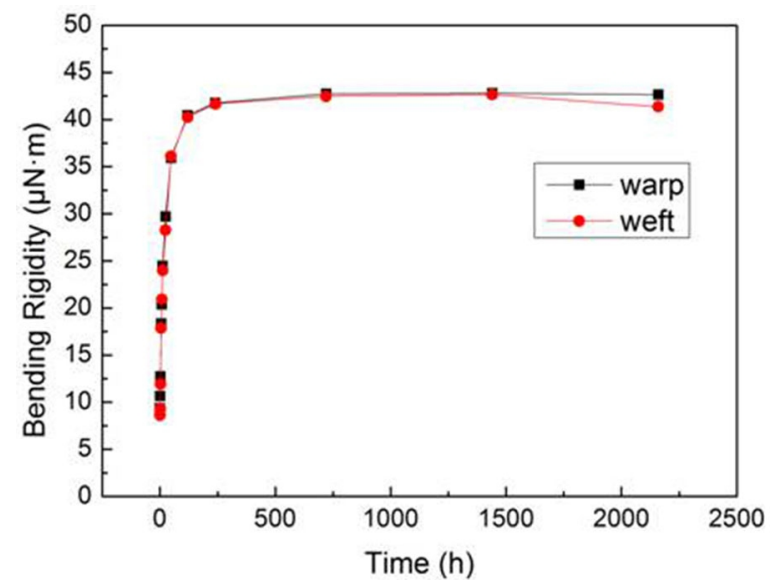

Figure 3. the variation of warp/weft bending rigidity with time

The bending rigidity of fabric with blood changes over time is shown in figure 3 . It can be seen that the trends of the variation of warp and weft bending rigidity with time are the same and the variation process can be mainly divided into three parts. Before 48 hours, the bending rigidity of fabric increases largely over time and the slope of the bending rigidity versus time curve is very high at initial time. From 48 hours to 240 hours, the variation of the bending rigidity over time reduces but the bending rigidity still increases obviously. After 240 hours, there is no significant change in bending rigidity. At last, the weft bending rigidity presents a slightly decrease after 1440 hours.

Blood is a kind of mixture, including volatile components and non-volatile components. When blood is spattered on fabric, the blood spreads quickly and is absorbed into the cotton plain woven fabric. The blood disperses among fibers and yarns which changes the friction between yarns and fibers. The volatile components of blood volatilizes continuously and the blood becomes denser. The denser blood makes the yarns and fibers difficult to move relatively. When the blood is dry, the dry blood acts as a adhesive substance, which binds the fibers and yarns tightly. So with the changes of the mechanical properties of blood, the bending rigidity of fabric changes. When the blood is dry and becomes solid blood clot, there is almost no change in the mechanical property of blood stains, so there is no obvious variation of the bending rigidity over time. As time goes on, the cohesiveness of dry blood stains become weak because of the weathering and microbial decomposition.

At the initial stage from 0 hour to 48 hours, the bending rigidity of fabric increases fast. From the 48 hours to 240 hours, the increase of bending rigidity becomes slowly. Figure 4 is the enlarged view of the front section of the curve showed in figure 3. It can be seen that from 0 to 120 hours the curve keeps a monotonic increase and the age of blood can be distinguished and speculated according to the bending rigidity of blooded fabric. Consequently, the age of blood can be effectively determined through testing the bending rigidity of blooded fabric if the age of blood is not over five days.

Through fitting the warp bending rigidity versus time curve, we obtained the fitted Equation (2).

$$
\begin{aligned}
& \mathrm{B}_{\mathrm{warp}}=7.231 \cdot \ln (0.612 * \mathrm{~T}+1)+9.37 \\
& \mathrm{~B}_{\text {warp }}: \text { warp bending rigidity, }[\mu \mathrm{N} \cdot \mathrm{m}] \\
& \mathrm{T} \text { : time, [hour] }
\end{aligned}
$$




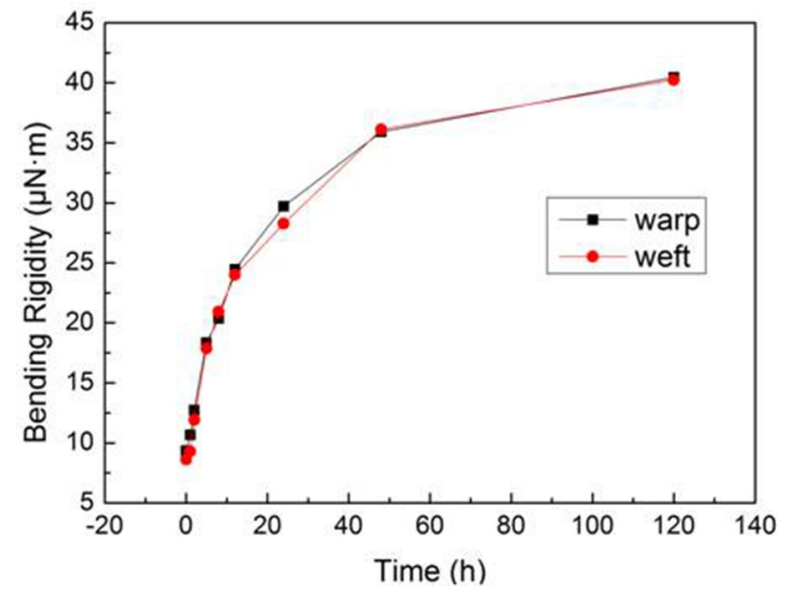

Figure 4. Magnification of the front section of the fabric bending rigidity

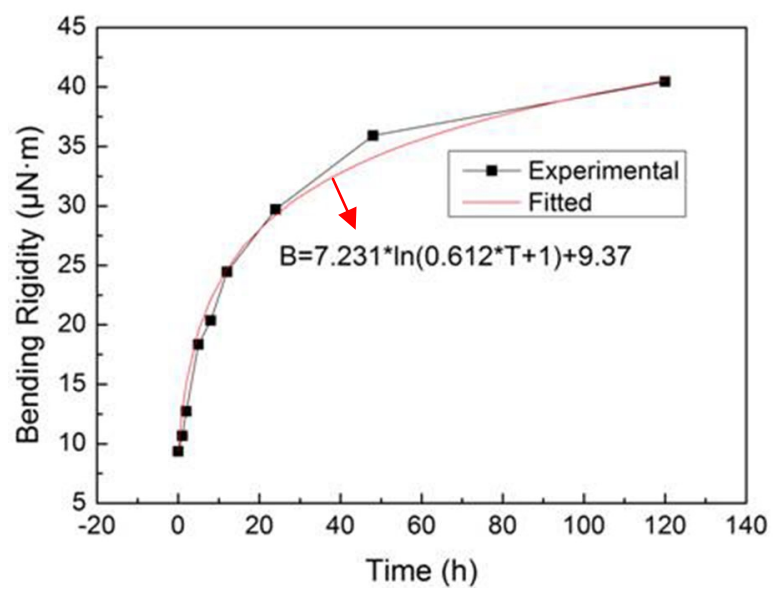

Figure 5. Fitted curve of the warp bending rigidity versus time curve

Figure 5 shows that the fitted curve is in good agreement with the experimental curve. The age of blood can be speculated through testing the bending rigidity. And this method is valid within 5 days and easy to operate.

\section{Conclusions}

The bending rigidity is an important mechanical property of fabric. The yarn stiffness, fabric tightness, fabric structure and the interaction between yarns influence the bending rigidity of fabric a lot. Blood changes the interaction between yarns and interaction between fibers. Consequently, the bending rigidity of blooded fabric is different. And the variation of mechanical property of blood over time reflects the age of blood. Experiments on the bending rigidity of blooded fabric have been done and several conclusions have been obtained as follows:

1) Blood influences the bending rigidity of cotton plain fabric obviously. No matter the warp direction or the weft direction, the influence of blood on the bending rigidity is the same.

2) The bending rigidity of blooded fabric changes obviously with time and the changing process can be mainly divided into three parts. Before 48 hours, the bending rigidity of fabric increases fast. From 48 hours to 240 hours, the variation of the bending rigidity reduces but the bending rigidity still increases obviously. After 240 hours, there is no significant change in bending rigidity.

3) Through testing the bending rigidity of blooded fabric, the age of blood can be effectively speculated if the age of blood is not over 5 days.

\section{Acknowledgements}

This work is supported by the Opening Project of Shanghai Key Laboratory of Crime Scene Evidence.

\section{References}

[1] Bremmer, R.H., et al., Forensic Sci Int, 2011. 206(1-3): p. 166-71.

[2] Edelman, G., T.G. van Leeuwen, and M.C. Aalders, Forensic Sci Int, 2012. 223(1-3): p. 72-7.

[3] Anderson, S., et al., Forensic Sci Int, 2005. 148(1): p. 37-45.

[4] Strasser, S., et al., Forensic Sci Int, 2007. 170(1): p. 8-14.

[5] Matsudaira, M., Y. Tan, and Y. Kondo, Journal of the Textile Institute, 1993: p. 376-386.

[6] Lammens, N., et al., Textile Research Journal, 2014. 84(12): p. 1307-1314.

[7] Moghassem, A.R., Fibers and Polymers, 2012. 13(2): p. 237-243. 